\title{
(-)-Epigallocatechin-3-gallate Down-regulates Doxorubicin-induced Overexpression of P-glycoprotein Through the Coordinate Inhibition of PI3K/Akt and MEK/ERK Signaling Pathways
}

\author{
HANA SATONAKA ${ }^{1}$, KUMIKI ISHIDA $^{1}$, MIHO TAKAI $^{1}$, RYOJI KOIDE $^{1}$, RYOTA SHIGEMASA $^{1}$, JUN UEYAMA $^{1}$, \\ TETSUYA ISHIKAWA ${ }^{1}$, KAZUHIKO HAYASHI ${ }^{2}$, HIDEMI GOTO $^{2}$ and SHINYA WAKUSAWA ${ }^{1}$ \\ ${ }^{1}$ Division of Medical Laboratory Sciences, Department of Radiological and Medical Laboratory Sciences, \\ Nagoya University Graduate School of Medicine, Nagoya, Japan; \\ ${ }^{2}$ Division of Gastroenterology, Department of Internal Medicine, \\ Nagoya University Graduate School of Medicine, Nagoya, Japan
}

\begin{abstract}
Background/Aim: (-)-Epigallocatechin-3-gallate (EGCG) has been indicated to regulate the function of $P$ glycoprotein $(P-g p)$, which is a drug transporter encoded by the MDR1 (ABCB1) gene. P-gp expression is induced by doxorubicin (DOX). We aimed to clarify the mechanisms and inhibitory effects of EGCG on DOX-induced P-gp expression in HepG2 cells. Materials and Methods: Rhodamine 123 (Rho123) was used for P-gp substrate. Western blotting and polymerase chain reactions (PCRs) were conducted using specific antibodies and primer sets. Results: The DOXpretreated cells accumulated a significantly decreased amount of Rho123), than control cells; however, the cells pretreated with EGCG and DOX, in combination, accumulated Rho123 more than DOX-pretreated cells. DOX induced the overexpression of MDRl $m R N A$ and increased the phosphorylation of Akt, ERK1/2, p38 MAPK and JNK. EGCG significantly inhibited the phosphorylation of Akt and ERK. The DOX-induced $P$-gp overexpression was partially suppressed by an inhibitor of MEK1/2 (U0126), but not by a PI3K inhibitor (LY294002). Interestingly, the expression of $P$-gp was synergistically inhibited by combined treatment of U0126 with LY294002 and also inhibited by an mTORC1 inhibitor, rapamycin. Conclusion: EGCG inhibited DOX-induced
\end{abstract}

This article is freely accessible online.

Correspondence to: Shinya Wakusawa, Division of Medical Laboratory Sciences, Department of Radiological and Medical Laboratory Sciences, Nagoya University Graduate School of Medicine, 1-1-20 Daiko-minami, Nagoya 461-8673, Japan. Tel: +81 527191558, e-mail: wakusawa@met.nagoya-u.ac.jp

Key Words: EGCG, P-glycoprotein, MEK/ERK, PI3K/Akt, doxorubicin. overexpression of $P$-gp through the coordinate inhibitory action on MEK/ERK and PI3K/Akt signaling pathways.

(-)-Epigallocatechin gallate (EGCG) is the most abundant component of tea polyphenols having various profitable properties, including anti-oxidative activity and cancerpreventive effect (1). In addition, concerning antitumor drug resistance, EGCG has been shown to decrease doxorubicin (DOX) resistance in a human carcinoma xenograft model mice (2) and inhibit the transport function of P-glycoprotein (P-gp), an export drug transporter causing antitumor multidrug resistance (MDR) in tumor cells (3-5).

Human P-gp is a plasma membrane protein of $170 \mathrm{kDa}$ encoded by the MDRl $(A B C B 1)$ gene. The expression of $M D R l$ gene and $\mathrm{P}$-gp is affected by various chemical substances and physico-chemical stress (5-7). We have previously suggested that the $\mathrm{P}$-gp level was up-regulated through the activation of protein kinase $\mathrm{C} /$ nuclear factor- $\mathrm{kB}$ $(\mathrm{PKC} / \mathrm{NF}-\mathrm{kB})$ in rats with streptozotocin-induced diabetes (8). Further, the expression of $M D R l$ gene has been also shown to be induced by PI3K/Akt/NF-kB signaling $(9,10)$.

EGCG has been shown to activate ERK and PI3K/Akt signaling and induce antioxidant enzymes in human mammary epithelial cells (11). Contrarily, it has been shown that EGCG inhibits the PI3K/Akt system in fibroblast cells (12) and the phosphorylation of ERK and Akt kinases in epidermal growth factor (EGF)-stimulated cells (13). More recently, accumulating evidence indicates that EGCG can inhibit PI3K/Akt and MAPK signaling systems (14-17).

From these backgrounds, in the present study, we aimed to clarify whether EGCG could suppress MDR and inhibit the induction of P-gp overexpression by DOX through inhibition of PI3K/Akt and ERK/MAPK signal transduction systems. 


\section{Materials and Methods}

Chemicals. EGCG, DOX, rhodamine 123 (Rho123), LY294002 and SB202190 were purchased from Sigma Japan (Tokyo, Japan). SP600125 was purchased from Biomol (Plymouth Meeting, PA, USA). U0126 was purchased from Cayman Chemical (Ann Arbor, MI, USA). Rapamycin was purchased from LC Laboratories (Woburn, MA, USA).

Cell culture. Human hepatoma HepG2 cells (RIKEN, Tsukuba, Japan) were cultured in Dulbecco's modified Eagle's medium (Sigma) supplemented with $5 \%(\mathrm{v} / \mathrm{v})$ heat-inactivated fetal calf serum (BioWest, Nauille, France), $100 \mathrm{U} / \mathrm{ml}$ of penicillin (Invitrogen Japan, Tokyo, Japan), $100 \mu \mathrm{g} / \mathrm{ml}$ of streptomycin (Invitrogen) and $0.25 \mu \mathrm{g} / \mathrm{ml}$ of amphotericin B (Invitrogen) in $35-\mathrm{mm}$ plastic dishes under $5 \% \mathrm{CO}_{2}$ at $37^{\circ} \mathrm{C}$ until semi-confluent. Then, varying concentrations of agents were added to the culture medium and the cells cultured for the designated periods. DOX and EGCG were dissolved in dimethyl sulfoxide (DMSO; Sigma) at an appropriate concentration and added to the culture medium. The concentration of DMSO was adjusted to $0.1 \%(\mathrm{v} / \mathrm{v})$ of the culture medium in each group.

Rho 123 accumulation assay. Rho123 was dissolved in DMSO and added to the cell culture medium at $3 \mu \mathrm{M}$. Cells were incubated for $30 \mathrm{~min}$ at $37^{\circ} \mathrm{C}$ and washed 3 times with phosphate-buffered saline (PBS, pH 7.4). Then, intracellular Rho123 was extracted with $1 \mathrm{ml}$ of ethanol and the fluorescence intensity was measured with ARVO MX multilabel reader (PerkinElmer, Waltham, MA, USA) with excitation and emission wave-lengths of $485 \mathrm{~nm}$ and $535 \mathrm{~nm}$, respectively.

Reverse transcription-polymerase chain reaction (RT-PCR). Total RNA was isolated using TRIzol Reagent (Invitrogen). Then, cDNA was prepared by incubation of 0.5-1.0 $\mu \mathrm{g}$ of the RNA with RNA reverse transcriptase (ReverTra Ace; TOYOBO, Tokyo, Japan) in $20 \mu \mathrm{l}$ of reaction buffer according to the ReverTra Ace data sheet. PCR was carried out with 0.5 units of Taq DNA polymerase (Blend Taq; TOYOBO) in $25 \mu \mathrm{l}$ of PCR solution using Thermal Cycler PxE (Thermo Fisher Scientific, Waltham, MA, USA). PCR primers for $M D R 1$ and glyceraldehyde-3-phosphate dehydrogenase (GAPDH) were synthesized by Hokkaido System Science. A primer set of MDR1 and GAPDH genes were as follows; MDR1 forward: 5'AAGCTTAGTACCAAAGAGGCTCTC-3', MDR1 reverse: 5'GGCTAGAAACAATAGTGAAAACAA-3', GAPDH forward: 5'ACCACAGTCCATGCCATCAC-3', GAPDH reverse: 5'TCCACCACCCTGTTGCTGTA-3'. The optimum cycle numbers of PCR were 30 to 35 cycles for MDR1 and 20 to 25 cycles for $G A P D H$. The PCR products $(10 \mu \mathrm{l})$ were electrophoresed on a $1.5 \%$ agarose gel and visualized with ultraviolet light and ethidium bromide. Densitometry analysis of the images was performed using ImageJ for Windows supplied by National Institutes of Health (Bethesda, MD, USA).

Western blot analysis of P-gp, ERK1/2, p38 MAPK, JNK and Akt. Cells $\left(1 \times 10^{4}\right)$ were solubilized in $20 \mu \mathrm{l}$ loading dye containing $125 \mathrm{mM}$ Tris (pH6.8, Sigma), 5\% sodium dodecyl sulfate (Sigma), 40\% urea (Sigma), $0.1 \mathrm{mM}$ sodium orthovanadate (Sigma), 0.2 M dithiothreitol (Sigma) and the sample was isolated by sodium dodecyl sulfate (SDS)polyacrylamide gel electrophoresis $(8,10 \%$ gel) and transferred onto a Hybond enhanced chemiluminescence (ECL) Nitrocellulose membrane
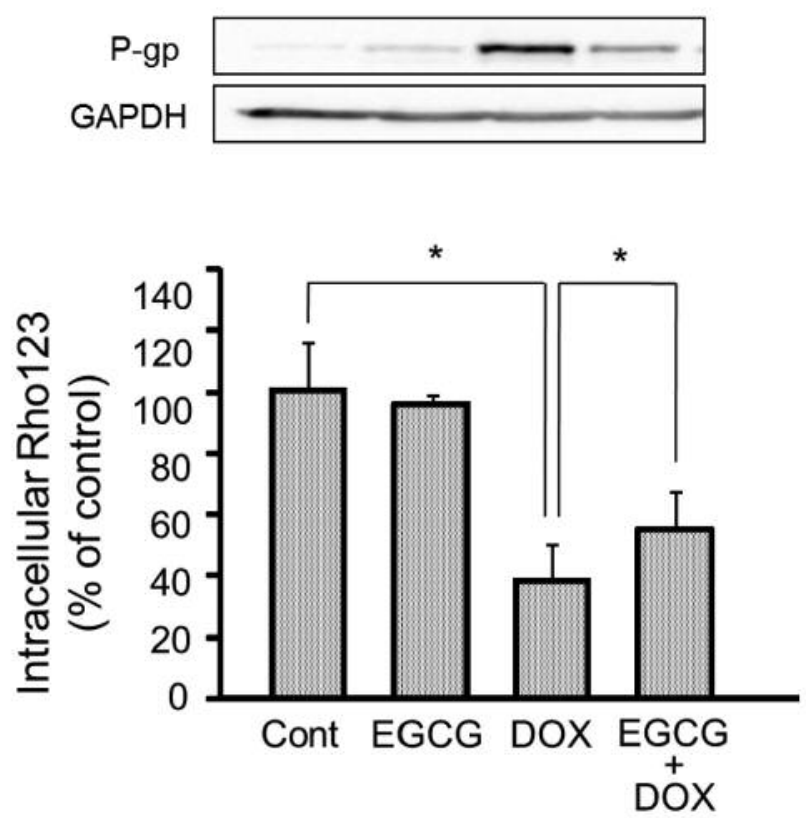

Figure 1. Preventive effects of (-)-epigallocatechin-3-gallate (EGCG) on the uptake of rhodamine 123 (Rho123) and P-glycoprotein (P-gp) level in the doxorubicin (DOX)-treated HepG2 cells. Cells were exposed to $3 \mu M D O X$ in the absence or presence of $50 \mu M E G C G$ for $24 \mathrm{~h}$. The cells were subsequently cultivated in fresh medium for $24 \mathrm{~h}$ and then Rho123 uptake was determined fluorometrically. A typical set of P-gp Western blot of the cells is shown in the panel. ${ }^{*} p<0.05$. Cont, Control; $G A P D H$, glyceraldehyde-3-phosphate dehydrogenase.

(GE Healthcare, Tokyo, Japan). After blocking with skim milk, the membrane was treated with primary antibodies.

P-gp was detected with the C219 anti-MDR1 P-gp mouse monoclonal antibody (Gene Tex, Irvine, CA, USA) as a primary antibody and horseradish-labeled goat anti-mouse $\mathrm{IgG}$ antibody (Santa Cruz Biotechnology, Dallas, CA, USA) as a secondary antibody. Total and phosphorylated ERK1/2, as well as p38 MAPK were detected with anti-ERK1/2, anti-phospho-ERK1/2, anti-38 MAPK and anti-phospho-p38 MAPK (Thr180/Tyr182) (Cell Signaling Technology, Danvers, MA, USA), respectively, as primary antibodies and horseradish-labeled goat anti-rabbit IgG antibody (Cell Signaling Technology) as a secondary antibody.

Western blot analysis of Akt was conducted according to previous reports (18).

Specific antibodies for GAPDH (Sigma) and $\beta$-actin (Medical \& Biological Laboratories, Aichi, Japan) were used to indicate loading standards.

The specific immunoreactive band was detected using Immobilon Western Detection Reagents (Merk Millipore, Bedford, MA, USA) and a luminescence imager (Light-Capture II; ATTO, Tokyo, Japan). The cellular protein concentration was determined using a DC protein assay kit (Bio-Rad Laboratories, Hercules, CA, USA).

Statistical analysis. Data are expressed as the mean \pm standard deviation (S.D.). Statistical significance was determined by a oneway analysis of variance (ANOVA) and Holm's multiple-comparison 

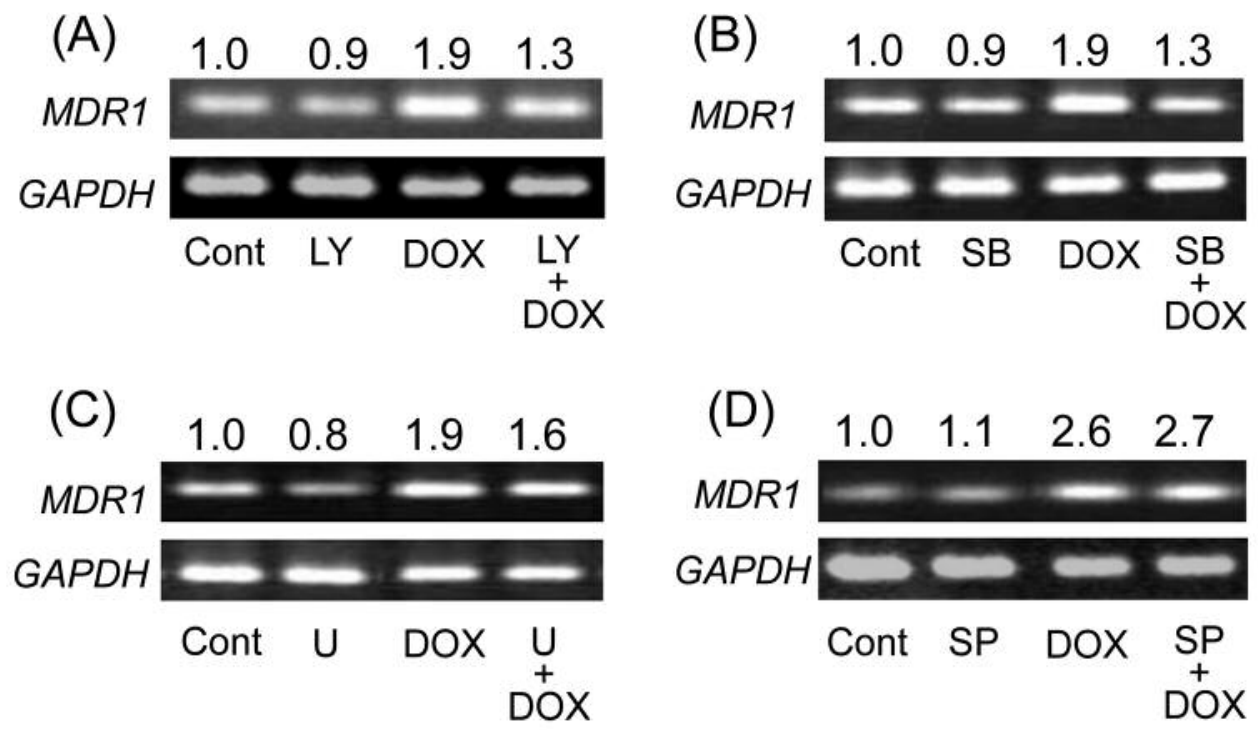

Figure 2. Effects of LY294002, SB202190, U0126 and SP600125 on doxorubicin (DOX)-induced up-regulation of multidrug resistance 1 (MDR1) gene in HepG2 cells. HepG2 cells were exposed to $3 \mu M$ DOX in the presence or absence of LY294002 (LY; $10 \mu M), S B 202190$ (SB; $3 \mu M), U 0126$ $(U ; 15 \mu M)$ and SP600125 (SP; $20 \mu M)$ for 20-24 h. Then, reverse transcription-polymerase chain reactions (RT-PCRs) of MDR1 and GAPDH were conducted using total RNA extracted from the cells. The photograph is typical of three independent experiments. Cont, Control.

test. All $p$-values $<0.05$ were considered statistically significant. All statistical analyses were performed using R Commander Plug-in for the EZR (Easy R) Package (RcmdrPlugin.EZR) (19).

\section{Results}

Preventive effect of EGCG on DOX-induced overexpression of $P$-gp and intracellular Rho123 accumulation. Rho123 is a useful substrate of P-gp for the evaluation of export function of P-gp, although it is not completely specific for P-gp (20-22). Since the expression of $\mathrm{P}-\mathrm{gp}$ and $M D R 1$ gene is induced by DOX as we have previously reported (22), we investigated the preventive action of EGCG against DOX-induced overexpression of P-gp and Rho123 accumulation in the cells. As shown in Figure 1, DOX-induced P-gp overexpression was apparently decreased by EGCG treatment. In relation to the induction of P-gp, the uptake of Rho123 was significantly decreased by DOX pretreatment. The uptake was significantly recovered in the cells pretreated with EGCG and DOX compared with the cells pretreated with DOX alone. These results indicated that EGCG had a potential of preventing DOX-induced overexpression of $\mathrm{P}$-gp and recovering intracellular accumulation of P-gp substrate.

Effects of cell signaling inhibitors on DOX-induced overexpression of MDRI $m R N A$. To clarify the mechanism of DOX-induced MDR1 expression, we investigated the effects of some cell signaling inhibitors for $M D R I$ mRNA expression in HepG2 cells. First, we examined whether DOX-induced MDR1 up-regulation is inhibited by LY294002 (a PI3K inhibitor). As shown in Figure 2A, LY294002 partially inhibited DOX-induced increase in the MDR1 mRNA level, while LY294002 did not affect the basal expression level.

There is contradictory evidence regarding the involvement of the p38 MAPK pathway in MDRl gene expression. Namely, Barancik et al. (23) and Katayama et al. (24) have reported that SB202190 (a p38 MAPK inhibitor) does not influence $M D R 1$ gene expression; Lu et al. (15), however, have recently indicated that SB203580 down-regulates both the P-gp protein level and MDR1 mRNA level in A2780/Taxol cells. In the present study, SB202190 (10 $\mu \mathrm{M})$ significantly suppressed the DOX-induced up-regulation of the MDRI gene, while SB202190 did not affect the basal expression level (Figure 2B). This indicated that the p38 MAPK pathway influenced DOX-induced $M D R 1$ gene expression.

We also investigated the effects of two other inhibitors on MAPK signaling. As shown in Figure 2C, U0126 (a MEK1/2 inhibitor) slightly decreased DOX-induced expressions of MDR1 mRNA but SP600125 (a c-Jun N-terminal kinase (JNK) inhibitor) did not influence its level (Figure 2D).

From these results, it was suggested that DOX induced MDR1 mRNA overexpression through PI3K signaling pathway and p38 MAPK signaling pathway in HepG2 cells. MEK/ERK signaling pathway may also contribute to the DOX-induced overexpression of MDRI mRNA; however, JNK signaling pathway does not seem to concern the induction. 
(A)
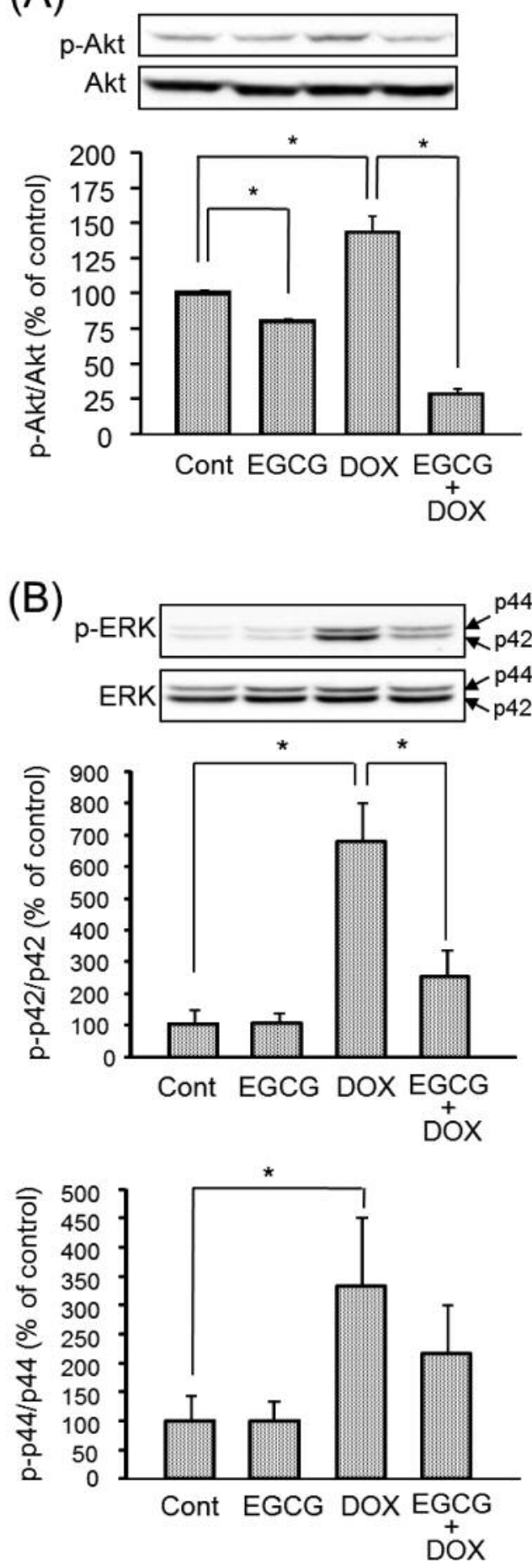

Effects of EGCG and DOX on the phosphorylation of Akt, $E R K 1 / 2$ and $p 38$ MAPK. To clarify the mechanism of the inhibitory action of EGCG against DOX-induced overexpression of MDR $1 \mathrm{mRNA}$, we examined the effect of
(C)

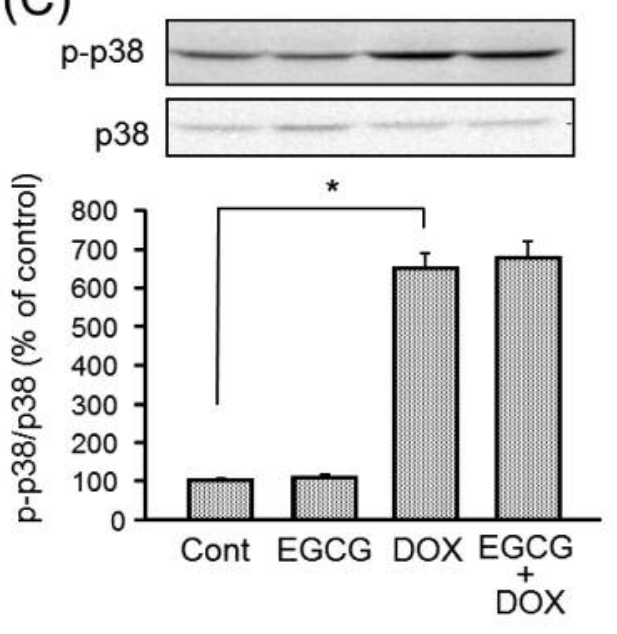

Figure 3. Effects of (-)-epigallocatechin-3-gallate (EGCG) on the phosphorylation of Akt, ERK, and p38 MAPK in doxorubicin (DOX)treated HepG2 cells. HepG2 cells were exposed to $3 \mu M D O X$ in the presence or absence of 50 $\mathrm{MM}$ EGCG for 20-24 h. Then, whole-cell lysates were used for western blot analysis of phosphorylated Akt ( $p$ $A k t)$ (A), phosphorylated ERK (p-ERK) (B) and phosphorylated p38 MAPK (p-p38) (C) as described in Materials and Methods. Each total protein level was also detected with a specific antibody. The photograph is typical of three independent experiments. Column graph data are expressed as the mean \pm S.D. $(n=3)$. *p<0.05. Cont, Control.

EGCG on the phosphorylation of Akt, ERK1/2 (p44/p42 MAPK) and p38 MAPK. As shown in Figure 3A, DOX significantly increased the phosphorylation of Akt at Ser473 , as was shown previously $(25,26)$, and EGCG strongly inhibited the phosphorylation of Akt without affecting the total Akt level. As for ERK1/2, DOX significantly increased the phosphorylation of ERK1/2, as previously demonstrated (27). EGCG strongly inhibited the DOX-induced phosphorylation of $\mathrm{p} 42$ molecule and had a tendency to suppress DOX-induced phosphorylation of p44 without affecting the total ERK1/2 levels (Figure 3B). As for p38 MAPK, DOX induced the phosphorylation of p38 MAPK (at Thr180/Tyr182) as reported previously $(27,28)$ but EGCG did not affect the basal and DOX-induced phosphorylation of p38 MAPK and the total p38 MAPK level in HepG2 cells (Figure 3C). From these results, it was indicated that the inhibition of the PI3K/Akt and ERK signaling pathways by EGCG contributed to prevent the DOX-induced upregulation of MDRI mRNA level.

Effect of rapamycin on P-gp expression in HepG2 cells exposed to DOX. Recently, it has been indicated that EGCG has an inhibitory property against mTOR, as well as PI3K (17). Therefore, we investigated the effect of rapamycin on P-gp level as this agent inactivates mammalian target of 


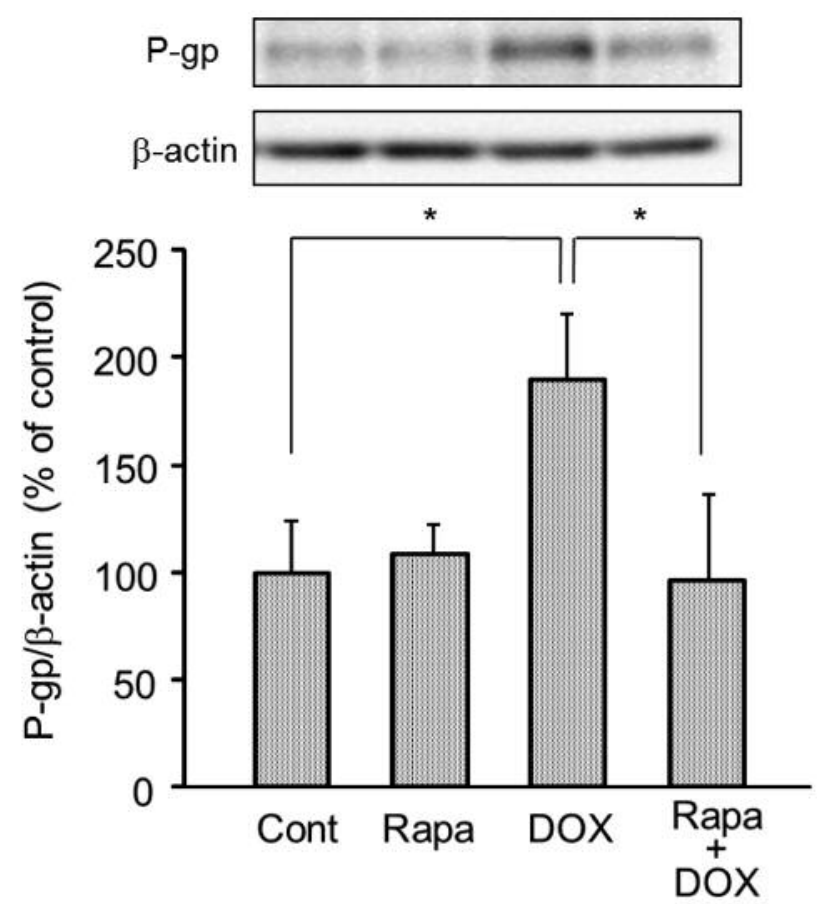

Figure 4. Effects of rapamycin on doxorubicin (DOX)-induced overexpression of P-glycoprotein ( $P$-gp) in HepG2 cells. HepG2 cells were exposed to $3 \mu \mathrm{M} D O X$ in the presence or absence of $10 \mathrm{nM}$ rapamycin for $24 \mathrm{~h}$. Then, western blot analysis of P-gp was conducted as described in Materials and Methods. The photograph is typical of three independent experiments. Column graph data are expressed as the mean \pm S.D. $(n=3) . * p<0.05$. Cont, Control.

rapamycin complex 1 (mTORC1). In the present study, rapamycin significantly inhibited the induction of P-gp by DOX (Figure 4). Since mTORC1 mediates a signal of PI3K/Akt pathway (29), we speculated that the activation of PI3K/Akt signaling by DOX and the inhibition of it by EGCG, seen in Figure 3A, might contribute to the regulation of P-gp overexpression.

Effects of the inhibitors for PI3K/Akt and MEK/ERK signaling pathways on P-gp expression in HepG2 cells exposed to DOX. As shown in Figures 2 and 3, the inhibitors for PI3K/Akt and MEK/ERK signaling pathways decreased the induction of MDRI mRNA and EGCG blocked DOXinduced activation of PI3K/Akt and MEK/ERK signaling. Further, rapamycin inhibited DOX-induced P-gp expression (Figure 4). Then we examined the effect of inhibitors for $\mathrm{PI} 3 \mathrm{~K} / \mathrm{Akt}$ and MEK/ERK signaling pathways on DOXinduced overexpression of P-gp (Figure 5). U0126 significantly inhibited DOX-induced P-gp overexpression, as well as MDR1 mRNA. However, LY294002 did not affect

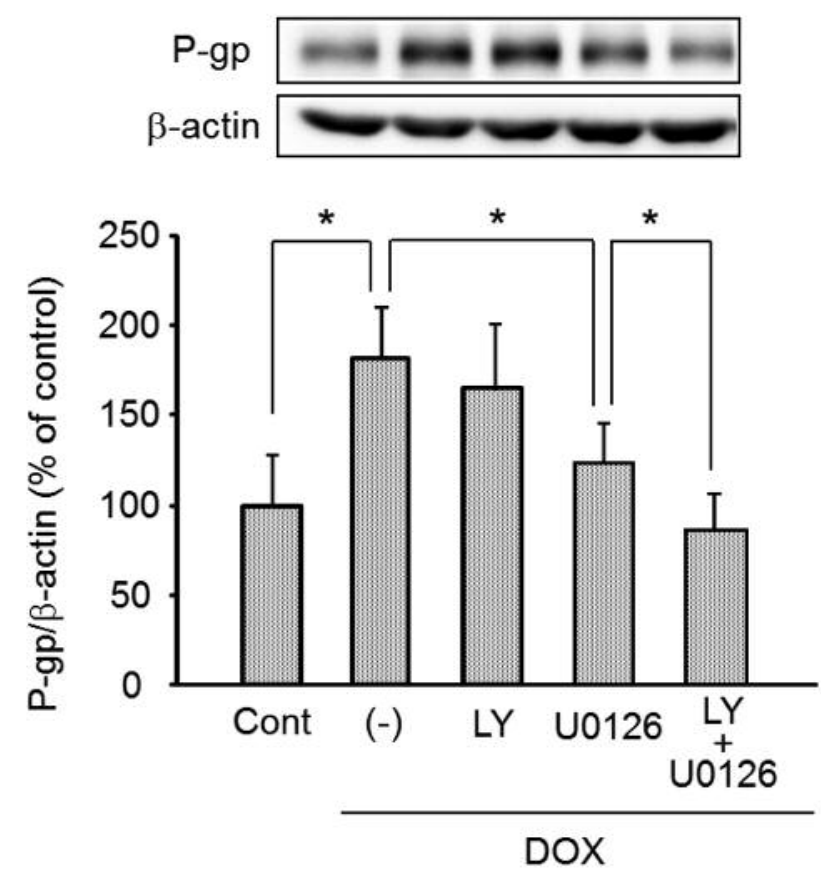

Figure 5. Effects of a combination of LY294002 with U0126 on Pglycoprotein ( $P$-gp) expression in HepG2 cells exposed to doxorubicin (DOX). HepG2 cells were exposed to $3 \mu M D O X$ in the presence or absence of LY294002 (LY, $10 \mu M), U 0126(15 \mu M)$ and the combination of the two inhibitors for 20-24 h. Then, whole cell lysates were used for Western blot analysis of P-gp as described in Materials and Methods. The photograph is typical of three independent experiments. Column graph data are expressed as the mean \pm S.D. $(n=3)$. ${ }^{*} p<0.05$. Cont, Control.

the expression of P-gp, while wortmannin also did not affect the DOX-induced overexpression of P-gp (data not shown). Interestingly, however, P-gp level was synergistically inhibited by the co-application of the inhibitors for PI3K/Akt and MEK/ERK signaling pathways. From these results, it was indicated that the dual inhibition of PI3K/Akt and MEK/ERK signaling pathways can effectively regulate the overexpression of P-gp and that PI3K/Akt signaling and MEK/ERK signaling pathways would coordinately regulate the expression of P-gp.

\section{Discussion}

Green tea polyphenol, containing more than 65\% EGCG, has been shown to sensitize MDR of KB-A-1 cells when inoculated to nude mice (30). EGCG has been known to inhibit export function of $\mathrm{P}$-gp and to reverse DOX resistance in vivo $(2,3,31)$, while a recent study by Cheng et al. (32) showed EGCG-based complex micelles with DOX effectively reducing cardiotoxicity in mice. 
In this study, we showed that EGCG selectively prevented DOX-induced P-gp overexpression at a dosage without influencing the basal expression of P-gp. Further, we showed that EGCG recovered Rho123 accumulation along with the suppression of P-gp overexpression when the cells were copretreated with DOX and EGCG. Thus, our results indicated that EGCG prevented the overexpression of P-gp and, thereby, inhibited enhancement of P-gp-mediated extrusion of its substrate.

In the present study, to clarify the signaling pathway concerning the inhibitory effect of EGCG on DOX-induced $M D R 1$ gene expression, we first evaluated the effects of specific kinase inhibitors on DOX-induced MDR1 mRNA expression in HepG2 cells. Regarding the role of PI3K/Akt signaling pathway, our results indicated that MDRl gene overexpression by DOX was partially inhibited by a PI3K/Akt inhibitor (Figure 2A). However, a PI3K/Akt inhibitor did not affect P-gp overexpression (Figure 5). Although EGCG inhibits DOX-induced Akt phosphorylation (Figure3A), the inhibition itself may scarcely contribute to the inhibition of P-gp overexpression in DOX-treated HepG2 cells. This phenomenon may indicate that MDRl mRNA overexpression is not enough for inducing $\mathrm{P}$-gp overexpression through the activation of PI3K/Akt.

It has already been reported an increase in phospho-ERK levels in DOX-treated HepG2 cells (33). In this study, we also showed that DOX induced activation of MEK/ERK pathway in HepG2 cells and that U0126, a MEK/ERK inhibitor, inhibited P-gp overexpression (Figure 5) and MDRl mRNA (Figure 2C). Further, EGCG significantly inhibited DOXinduced phosphorylation of p42 ERK molecule (Figure 3B). These findings may suggest that MEK/ERK pathway significantly contributes to the overexpression of P-gp induced by DOX. Further, it may be suggested that DOX-stimulated MEK/ERK signaling may positively and post-transcriptionally control P-gp expression. In addition, it was indicated that the inhibitory action of EGCG against MEK/ERK pathway was implicated in the inhibition of P-gp overexpression.

P-gp expression was synergistically inhibited by the combined treatment with PI3K/Akt and ERK inhibitors (Figure 5). This evidence may indicate that DOX increases P-gp expression through co-activation of these kinases. Further, these signaling pathways may coordinately regulate the expression of P-gp. As EGCG inhibits PI3K/Akt and MEK/ERK signaling, EGCG would negatively regulate P-gp overexpression through dual inhibition of these signaling pathways in HepG2 cells, although further potential mechanisms remain to be clarified. As rapamycin, which is an inhibitor of mTOR, inhibited DOXinduced overexpression of P-gp (Figure 4) and EGCG reportedly has an inhibitory effect on mTOR (14), which is a down-stream effector of PI3K/Akt signaling system, cross-talk of PI3K/Akt and ERK signaling pathways (29) may be involved in the control of P-gp expression.

\section{Conclusion}

EGCG inhibited DOX-induced overexpression of P-gp. According to the evidence of DOX-induced overexpression of P-gp accompanied by co-activation of MEK/ERK and PI3K/Akt signaling cascades and the inhibition by EGCG of DOX-induced activation of these pathways, it can be suggested that EGCG has a profitable efficacy of regulating DOX-induced P-gp overexpression through the coordinate inhibition of PI3K/Akt and MEK/ERK signaling pathways.

\section{Conflicts of Interest}

All Authors declare that there are no conflicts of interest.

\section{Funding}

This research did not receive any specific grant from any funding agency of the public, commercial or not-for-profit sector.

\section{References}

1 Du GJ, Zhang Z, Wen XD, Yu C, Calway T, Yuan CS and Wang CZ: Epigallocatechin gallate (EGCG) is the most effective cancer chemopreventive polyphenol in green tea. Nutrients 4 : 1679-1691, 2012.

2 Zhang Q, Wei D and Liu J: In vivo reversal of doxorubicin resistance by (-)-epigallocatechin gallate in a solid human carcinoma xenograft. Cancer Lett 208: 179-186, 2004.

3 Jodoin J, Demeule $\mathrm{M}$ and Beliveau R: Inhibition of the multidrug resistance P-glycoprotein activity by green tea polyphenols. Biochim Biophys Acta 1542: 149-159, 2002.

4 Qian F, Wei D, Zhang Q and Yang S: Modulation of Pglycoprotein function and reversal of multidrug resistance by (-)epigallocatechin gallate in human cancer cells. Biomed Pharmacother 59: 64-69, 2005.

5 Chin KV, Tanaka S, Darlington G, Pastan I and Gottesman MM: Heat shock and arsenite increase expression of the multidrug resistance (MDR1) gene in human renal carcinoma cells. J Biol Chem 265: 221-226, 1990.

6 Thévenod F, Friedmann JM, Alice D, Katsen AD, Ingeborg A and Hauser IA: Up-regulation of multidrug resistance Pglycoprotein via nuclear factor- $\mathrm{kB}$ activation protects kidney proximal tubule cells from cadmium- and reactive oxygen species-induced apoptosis. J Biol Chem 275: 1887-1896, 2000.

7 Ziemann C, Bürkle A, Kahl GF and Hirsch-Ernst KI: Reactive oxygen species participate in mdr1b mRNA and P-glycoprotein overexpression in primary rat hepatocyte cultures. Carcinogenesis 20: 407-414, 1999.

8 Kameyama N, Arisawa S, Ueyama J, Kagota S, Shinozuka K, Hattori A, Tatsumi Y, Hayashi H, Takagi K and Wakusawa S: Increase in $\mathrm{P}$-glycoprotein accompanied by activation of protein kinase Calpha and NF-kappaB p65 in the livers of rats with streptozotocin-induced diabetes. Biochim Biophys Acta 1782: 355-360, 2008

9 Choi BH, Kim CG, Lim Y, Shin SY and Lee YH: Curcumin down-regulates the multidrug-resistance mdrlb gene by 
inhibiting the PI3K/Akt/NF kappa B pathway. Cancer Lett 259: 111-118, 2008.

10 García MG, Alaniz LD, Cordo Russo RI, Alvarez E and Hajos SE: PI3K/Akt inhibition modulates multidrug resistance and activates NF-kappaB in murine lymphoma cell lines. Leuk Res 33: 288-296, 2009.

$11 \mathrm{Na}$ HK, Kim EH, Jung JH, Lee HH, Hyun JW and Surh YJ: (-)Epigallocatechin gallate induces Nrf2-mediated antioxidant enzyme expression via activation of PI3K and ERK in human mammary epithelial cells. Arch Biochem Biophys 476: 171-177, 2008.

12 Zhang Q, Kelly AP, Wang L, French SW, Tang X, Duong HS, Messadi DV and Le AD: Green tea extract and (-)epigallocatechin-3-gallate inhibit mast cell-stimulated type I collagen expression in keloid fibroblasts via blocking PI-3K/AkT signaling pathways. J Invest Dermatol 126: 2607-2613, 2006.

13 Sah JF, Balasubramanian S, Eckert RL and Rorke EA: Epigallocatechin-3-gallate inhibits epidermal growth factor receptor signaling pathway. Evidence for direct inhibition of ERK1/2 and AKT kinases. J Biol Chem 279: 12755-12762, 2004.

14 Van Aller GS, Carso JD, Tang W, Peng H, Zhao L, Copeland RA, Tummino PJ and Luo L: Epigallocatechin gallate (EGCG), a major component of green tea, is a dual phosphoinositide-3kinase/mTOR inhibitor. Biochem Biophys Res Commun 406: 194-199, 2011.

15 Liu S, Li H, Chen L, Yang L, Li L, Tao Y, Li W, Li Z, Liu H, TangM, BodeAM, Dong Z and Cao Y: (-)-Epigallocatechin-3gallate inhibition of Epstein-Barr virus spontaneous lytic infection involves ERK1/2 and PI3-K/Akt signaling in EBVpositive cells. Carcinogenesis 34: 627-637, 2013.

16 He L, Zhang E, Shi J, Li X, Zhou K, Zhang Q, Le AD and Tang $\mathrm{X}$ : (-)-Epigallocatechin-3-gallate inhibits human papillomavirus (HPV)-16 oncoprotein-induced angiogenesis in non-small cell lung cancer cells by targeting HIF-1 $\alpha$. Cancer Chemother Pharmacol 71: 713-725, 2013.

17 Xiao J, Ho CT. Liong E, Nanji AA, Leung TM, Lau,TY, Fung ML and Tipoe GL: Epigallocatechin gallate attenuates fibrosis, oxidative stress, and inflammation in non-alcoholic fatty liver disease rat model through TGF/SMAD, PI3 K/Akt/FoxO1, and NF-kappa B pathways. Eur J Nutr 53: 187-199, 2014.

18 Arisawa S, Ishida K, Kameyama N, Ueyama J, Hattori A, Tatsumi Y, Hayashi H, Yano M, Hayashi K, Katano Y, Goto H, Takagi, $\mathrm{K}$ and Wakusawa S: Ursodeoxycholic acid induces glutathione synthesis through activation of PI3K/Akt pathway in HepG2 cells. Biochem Pharmacol 77: 858-866, 2009.

19 Kanda Y: Investigation of the freely available easy-to-use software 'EZR' for medical statistics. Bone Marrow Transplant 48: 452-458, 2013.

20 Efferth T, Löhrke H and Volm M: Reciprocal correlation between expression of P-glycoprotein and accumulation of rhodamine 123 in human tumors. Anticancer Res 9: 1633-1637, 1989.

21 Chaudhary PM and Roninson IB: Expression and activity of Pglycoprotein, a multidrug efflux pump, in human hematopoietic stem cells. Cell 66: 85-94, 1991.
22 Komori Y, Arisawa S, Takai M, Yokoyama K, Honda M, Hayashi K, Ishigami M, Katano Y, Goto H, Ueyama J, Ishikawa $\mathrm{T}$ and Wakusawa $\mathrm{S}$ : Ursodeoxycholic acid inhibits overexpression of P-glycoprotein induced by doxorubicin in HepG2 cells. Eur J Pharmacol 724: 161-167, 2014.

23 Barancík M, Bohácová V, Kvackajová J, Hudecová S, Krizanová $\mathrm{O}$ and Breier A: SB203580, a specific inhibitor of p38-MAPK pathway, is a new reversal agent of P-glycoprotein-mediated multidrug resistance. Eur J Pharm Sci 14: 29-36, 2001.

24 Katayama K, Yoshioka S, Tsukahar S, Mitsuhashi J and Sugimoto Y: Inhibition of the mitogen-activated protein kinase pathway results in the down-regulation of P-glycoprotein. Mol Cancer Ther 6: 2092-2102, 2007.

25 Alexia C, Bras M, Fallot G, Vadrot N, Daniel F, Lasfer M, Tamouza $\mathrm{H}$ and Groyer A: Pleiotropic effects of PI-3' kinase/Akt signaling in human hepatoma cell proliferation and drug-induced apoptosis. Ann N Y Acad Sci 1090: 1-17, 2006.

$26 \mathrm{Li} \mathrm{X,} \mathrm{Lu} \mathrm{Y,} \mathrm{Liang} \mathrm{K,} \mathrm{Liu} \mathrm{B} \mathrm{and} \mathrm{Fan} \mathrm{Z:} \mathrm{Differential} \mathrm{responses} \mathrm{to}$ doxorubicin-induced phosphorylation and activation of Akt in human breast cancer cells. Breast Cancer Res 7: R589-R597, 2005.

27 Niiya M, Niiya K, Shibakura M, Asaumi N, Yoshida C, Shinagawa K, TeshimaT, Ishimaru F, Ikeda $\mathrm{K}$ and Tanimoto M: Involvement of ERK1/2 and p38 MAP kinase in doxorubicininduced uPA expression in human RC-K8 lymphoma and NCIH69 small cell lung carcinoma cells. Oncology 67: 310-319, 2004.

28 Poizat C, Puri PL, Bai Y and Kedes L: Phosphorylationdependent degradation of p300 by doxorubicin-activated p38 mitogen-activated protein kinase in cardiac cells. Mol Cell Biol 25: 2673-2687, 2005.

29 Mendoza MC, Er EE and Blenis J: The Ras-ERK and PI3KmTOR pathways: Cross-talk and compensation. Trends Biochem Sci 36: 320-328, 2011.

30 Mei Y, Qian F, Wei D and Liu J: Reversal of cancer multidrug resistance by green tea polyphenols. J Pharm Pharmacol 56: 1307-1314, 2004.

31 Kitagawa S, Nabekura, T and Kamiyama S: Inhibition of Pglycoprotein function by tea catechins in KB-C2 cells. J Pharm Pharmacol 56: 1001-1005, 2004.

32 Cheng T, Liu J, Ren J, Huang F, Ou H, Ding Y, Zhang Y, Ma R, An Y, Liu J and Shi L: Green tea catechin-based complex micelles combined with doxorubicin to overcome cardiotoxicity and multidrug resistance. Theranostics 6: 1277-1292, 2016.

33 Choi J, Yip-Schneider M, Albertin F, Wiesenauer C, WangY and Schmidt CM: The effect of doxorubicin on MEK-ERK signaling predicts its efficacy in HCC. J Surg Res 150: 219-226, 2008.

Received April 12, 2017

Revised April 26, 2017

Accepted April 27, 2017 